\title{
Testing Two Theories for Generating Signed Networks Using Real Data
}

\author{
Patrick Doreian ${ }^{1}$ and Andrej Mrvar ${ }^{2}$
}

\begin{abstract}
Multiple social processes generate social network structures. We use relaxed structural balance, a generalization of classic structural balance, to facilitate a direct comparative test of two social psychological theories regarding network generation. One is structural balance theory. The other concerns differential popularity. These theories predict distinctive signed blockmodels. We use two well known empirical temporal signed data sets presenting an opportunity for comparing the two theories in terms of their predictions about blockmodel representations of these networks. The results provide strong support for differential popularity, differential disliking, and mutual disliking within a subset of actors. While there is evidence that structural balance was also operating, it seems the lesser process for the data used in these tests. We also examine the unequal distributions of receiving positive and negative ties. Both tend to become more unequal over time. Suggestions for future research are provided.
\end{abstract}

\section{Introduction}

Both social psychologists and social network analysts develop theories intended to help understand social processes in small social groups. To the extent that the former focus more on node-level (actor) characteristics while the latter are more attentive to the network structure as a whole, there is a tension between micro-level and macro-level phenomena (Robins and Kashima, 2008). Our focus here is on understanding processes that generate network structures. We provide comparative tests of two theories based on a simple assumption: social processes, if operative in small groups, leave traces of recognizable patterns of network ties. This comparative test is for signed networks. Our primary goal is disentangling the results from the operation of processes specified by two theories of social processes in groups. One is structural balance theory Heider $(1946,1958)$ The other concerns

\footnotetext{
1 Faculty of Social Sciences, University of Ljubljana and Department of Sociology, University of Pittsburgh, 2602 WWPH, Pittsburgh, PA 15260; pitpat@ pitt.edu

${ }^{2}$ Faculty of Social Sciences, University of Ljubljana, Kardeljeva pl. 5, 1000 Ljubljana, Slovenia; Andrej.Mrvar@fdv.uni-lj.si
} 
differential popularity, a process described by Feld and Elsmore (1984) under which some group members receive more positive ties than others. The detailed predictions of the two theories differ.

As Taylor (1970) notes, Heider was credited with the initial statement of structural balance theory. While we focus attention on the Heider variant of consistency theories, Newcomb (1961), Festinger (1957), Osgood and Tannenbaum (1955) and others (see Abelson et al., 1968) also formulated alternative consistency theories. We use Heider's approach because Cartwright and Harary's (1956) formal generalization of his theory laid formal foundations for analyzing signed social networks.

Feld and Elsmore (1984) drew a critical response from Hallinan (1984) regarding rival processes accounting for the unequal distributions in the receipt of signed ties in a group. Both papers considered rival theories about group processes by using distributions of particular triples of ties among trios of actors in the network of actors in the group.

Rather than use distributions of triple types, we examine the overall structure of a network using blocks located in signed blockmodels. Briefly, a blockmodel of a network is a simultaneous partition of both the actors and their social ties. The clusters of actors are called positions ${ }^{3}$. Using blockmodels delineating network structure provides an direct description of a network's overall structure.

The rest of this paper is organized as follows. Section 2 outlines substantive issues and Section 3 describes our data and methods. Section 4 presents our results and we conclude with a summary and discussion in Section 5.

\section{Theories about processes that generate network structures}

\subsection{Structural balance theory}

The intuitions of Heider's (1946) structural balance theory, formalized by Cartwright and Harary (1956), led to a sustained research effort of discerning the structure of signed networks (Doreian et al., 2005: Chapter 10). Key in this development was a remarkable 'structure theorem' coupling micro-processes (of actors forming and/or dropping signed ties) and the resulting macro-structure of the group. Signed ties are either positive (e.g. liking, loving, supporting) or negative (e.g. disliking, hating, opposing). For three actors, denoted by $p, o$ and $q$, in a signed network, the poq triple is made up of the ties $(p \rightarrow q),(q \rightarrow o)$ and $(\mathrm{p} \rightarrow \mathrm{o})$. The sign of every triple is the

\footnotetext{
3 A formal statement can be found in Doreian et al. (2005). Ferligoj et al. (2011) contains a rigorous informal statement about positional analysis in terms of positions and roles.
} 
product of its signed relations. A poq-triple is balanced if its sign is positive and imbalanced if the sign is negative ${ }^{4}$. There are four possible balanced triples and four imbalanced triples. A signed network is balanced if all of its poq-triples are balanced. Cartwright and Harary's main theorem states: the vertices of a balanced network can be partitioned into two positions where all of the positive ties are within positions and all of the negative ties are between members of different positions. This result links the micro-processes of tie formation and change within triads to a statement about the overall group structure for balanced networks. Davis (1967) noted human groups often have more than two mutually hostile subgroups. He generalized Cartwright and Harary's result by reconsidering one part of Heider's foundational statement: if all of the ties in a poq-triple were negative, the triple was imbalanced. Davis defined this all-negative triple as balanced. His result was: a 'clusterable' network ${ }^{5}$ has two or more positions where all the positive ties were within clusters and all of the negative ties were between actors in different positions. This also links micro-processes to the macro-structure of a group. A signed network is $k$-balanced if it has the above partition structure. For $k=2$ it is Cartwright and Harary's structure theorem. For $k>2$ it is the generalization.

Blockmodeling (see Breiger et al., 1975; Doreian et al., 2005) has techniques for partitioning network data into positions (containing actors) and blocks (of ties between positions). The location of an actor is the set of ties to and from all other actors in the group. These locations of actors are clustered to form the positions. For $n$ actors, the $n$ locations are partitioned into $k$ positions with $k$ is much smaller than $n$. A large network is reduced to a smaller image matrix with $k$ positions and $k^{2}$ blocks representing the essential network structure. Doreian and Mrvar (1996) noticed the theorems of Cartwright and Harary (1956) and Davis (1967) can be viewed as leading to statements of specific blockmodels. A positive block is one having only positive ties and null ties while a negative block has only negative ties and null ties. From the structure theorems, in a $k$-balanced network, the signed blockmodel has positive blocks on the main diagonal (top left to bottom right) and negative blocks off the main diagonal. If, for example, $k=4$ and structural balance is the only process operating, then the blockmodel implied by structural balance is simple to describe. The block pattern for four positions is:

\footnotetext{
4 This is expressed in folk aphorisms: "a friend of a friend is a friend", "a friend of an enemy is an enemy", "an enemy of a friend is an enemy" and "an enemy of an enemy is a friend". These have simple cognitive structures. As Mower White (1979) notes, simple cognitive structures are more likely than complex structures to exhibit balance. Also, "it is now recognized that if sentiment is restricted to the two values of positive and negative, balance is a simple implication of ordinary deductive logic (Montoya and Insko, 2008: 494)".

5 To prove this theorem, Davis used the concept of a semiwalk, an alternating sequence of vertices and arcs where the direction of the arcs is irrelevant. For pairs of actors between whom there exist one or more semiwalks, the sign for each of these semiwalks is the product of the signs of the arcs in the semiwalk. These signs are positive or negative. He defined a network as 'clusterable' if it had no semiwalks with a single negative arc.
} 
Positive Negative Negative Negative

Negative Positive Negative Negative

Negative Negative Positive Negative

Negative Negative Negative Positive

We refer to these as ideal blocks by location, call this blockmodel the Structural Balance blockmodel, and label it the 'SB Model'.

Regardless of the number of positions, every blockmodel predicted by structural balance has this generic (ideal) SB Model form. The number of positions, $k$, has to be determined as a part of fitting blockmodels. Empirically, it is unreasonable to expect a perfect correspondence between an ideal structure and an empirical structure. If structural balance is appropriate we would anticipate the SB Model but with some inconsistencies compared to the ideal structure.

Doreian and Mrvar (1996) took the form of the idealized blockmodels implied by structural balance and proposed a partitioning approach for establishing empirical blockmodel structure(s) of signed networks closest to the ideal form implied by the structural theorems. When empirical blockmodels do not fit exactly there are some inconsistencies between the empirical blockmodel and the ideal counterpart. These will take the form of some negative ties in positive blocks and some positive ties in negative blocks. The former are termed negative inconsistencies, the latter are positive inconsistencies. For a binary network (where the ties are +1 or -1 ), the total number of positive inconsistencies is denoted by $P$ and the total number of negative inconsistencies ${ }^{6}$ by $X$. A general measure of how

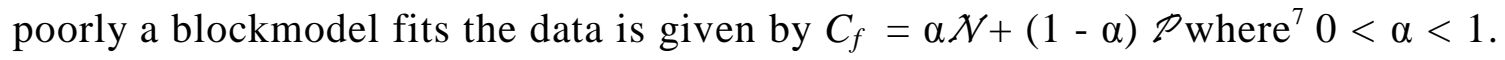
With $\alpha=0.5$, the two types of inconsistencies are weighted equally, a convention we use here. In essence, $C_{f}$ is the line index of imbalance proposed by Harary et al. (1965: 348-350). $C_{f}$ is a criterion function and the relocation clustering algorithm used by Doreian and Mrvar seeks optimal partition(s) minimizing this criterion function $^{8}$. Structural balance implies an SB Model.

\subsection{Differential popularity}

In the main, social scientists collecting sociometric data focused on unsigned data with only positive ties. Undoubtedly, such data are easier to collect. Also, one rationale for making comparisons of the distribution of triples in unsigned

\footnotetext{
6 If a network has weighted ties then $\mathrm{P}$ and $\mathrm{N}$, respectively, are the sums of positive and negative inconsistencies.

For $\alpha=1$, positive inconsistencies are ignored and negative inconsistencies are ignored for $\alpha=0$. Neither extreme weighting is useful when both positive and negative ties exist.

8 It is a local optimization method so finding the optimal partition(s) is not guaranteed. Brusco et al. (2011) established this algorithm has, thus far, identified all of the optimal partitions for signed networks up to 40 actors.
} 
networks, as used by Feld and Elsmore (1984) and by Hallinan (1984), is based on arguments of Davis and Leinhardt (1972) where signed graphs are 'converted' to unsigned counterparts. Rather than focus on signed ties (positive, null, and negative), attention was focused on mutual (M), null (N) and asymmetric (A) ties. Identifying clusters of positively connected actors, such as those among the positions of signed networks, was treated as evidence of a tendency towards clustering. Comparisons were then made of the distributions of the 16 possible triples involving $\mathrm{M}, \mathrm{A}$, and $\mathrm{N}$ ties. However, as using unsigned data handicaps any examination of balance theoretic ideas about signed networks, these efforts labored under a serious constraint: negative ties were excluded ${ }^{9}$. Feld and Elsmore (1984) focused primarily on transitivity. If $(p \rightarrow o)$ and $(o \rightarrow q)$ are present in an unsigned network then, under transitivity, the $(p \rightarrow q)$ tie will be present also. Empirically, there is a tendency towards transitivity in most unsigned networks with transitivity has regarded as a fundamental network process (Holland and Leinhardt, 1972; Wasserman and Faust, 1994: 243-248). Confirmation came with there being more transitive triples in a network than would be expected by chance. One key feature of Feld and Elsmore's argument is that some of the evidence for transitivity might be due to the operation of a process of differential popularity ${ }^{10}$. They provided some evidence in the form of distributions of poqtriples to support this claim. However, they were careful to not state differential popularity dominated transitivity. They suggested it could be a plausible generating process, one also creating some transitivity. In neutral terminology, transitivity and differential popularity are often confounded in empirical networks. When only one of them is considered, some of the support for it as the generating process will be spurious.

The idea of differential popularity extends straightforwardly to signed networks: some actors may be more popular and so receive more positive ties regardless of the presence of mutually hostile subgroups. If some members of a group are universally popular, then with $k=4$, the group structure, as a blockmodel, would be as follows if there were just two processes - structural balance and differential popularity - operating. An ideal blockmodel would look like:

\section{Positive Negative Negative Negative \\ Positive Positive Negative Negative \\ Positive Negative Positive Negative \\ Positive Negative Negative Positive}

\footnotetext{
9 We do not dispute the value of the highly productive work on triadic censuses for unsigned networks and their extension to exponential random graph models. But when structural balance is involved, we contend that both positive and negative ties must be included.

10 For example, given $p \rightarrow o$ and $o \rightarrow q$ as positive ties, if $p \rightarrow q$ exists then it can be viewed as being consistent with transitivity. It is consistent also with structural balance in a positive triple.
} 
Note the column of positive blocks on the left of this ideal blockmodel. Except for the top block, all of the positive blocks in the first column are inconsistent with structural balance (and are bolded for this reason). We call this ideal blockmodel a Structural $\underline{B}$ alance with Differential $\underline{P}$ opularity blockmodel and label it the SB_DP Model. If some additional actors are popular but not universally popular, an ideal blockmodel would look like:

\section{Positive Negative Negative Negative \\ Positive Positive Negative Negative \\ Positive Positive Positive Negative \\ Positive Positive Negative Positive}

The additional bolded blocks (in the second column of blocks) are also inconsistent with structural balance but consistent with differential popularity. This blockmodel is a variant of the SB_DP Model. There may be less extreme configurations where only some blocks in the left hand column are positive. There could be other subgroups receiving positive ties from members of other positions. These can be accommodated. For now, we focus on the SB_DP Model in our comparative tests.

Discriminating between these two theories can be done in a direct fashion. If structural balance operates, then the SB Model is appropriate. Further, if differential popularity is not operative, the SB Model would fit the data and not the SB_DP Model. But if the SB_DP Model is identified empirically, greater credibility is given to differential popularity. The partitioning algorithm of Doreian and Mrvar (1996) is useless for this comparative test: a SB Model is the only fitted blockmodel. However, thinking in terms of relaxing structural balance (Doreian and Mrvar, 2009) led to the creation of an algorithm appropriate for distinguishing these two models.

\subsection{Relaxed structural balance}

In responding to Feld and Elsmore (1984), Hallinan (1984) argued at least five substantive processes could generate transitivity in unsigned networks: differential expansiveness; reciprocity; differential popularity; clustering and cognitive (structural) balance. Although we do not focus on transitivity and consider signed networks, we accept the point of analyses of network data requiring recognition, and consideration, of multiple processes. Incorporating them for signed networks, when considering balance theoretic ideas, requires a generalization of structural balance. Reciprocal positive ties can be accommodated easily to the extent that 
they occur among within actors in the same position. But, if there is positive reciprocity between pairs of actors in different positions, this creates problems for structural balance: positive inconsistencies contribute to $C_{f}$. If this involves multiple pairs in two positions there will be corresponding positive blocks above and below the main diagonal. If there is reciprocation of negative ties between actors in different positions this will be consistent with structural balance. However, we need to consider subsets of actors who, as individuals, are mutually hostile towards each other. Their presence also contradicts structural balance because this implies a negative diagonal block ${ }^{11}$. If we add mutual dislike at the actor level for a set of actors - a "nest of vipers" in the colorful terminology of Hummert et al. (1990) - to differential popularity and structural balance then we would expect a structure approximating the following blockmodel:

\section{Positive Negative Negative Negative \\ Positive Positive Negative Negative \\ Positive Negative Positive Negative \\ Positive Negative Negative Negative}

Locating the diagonal negative block on the bottom right of the blockmodel appears arbitrary. But if there is a differential popularity process then it is reasonable to anticipate differential disliking implies negative ties are concentrated actors other than popular actors ${ }^{12}$. This is represented by a column of off-diagonal negative blocks on the right of this blockmodel. Further, if those that are more disliked also tend to dislike each other this implies a diagonal negative block. To capture this, we locate (and bold) a diagonal negative block at the bottom right hand side while recognizing that there could be more than one such block and they could appear anywhere on the diagonal. The column of off-diagonal negative blocks on the right is consistent with both structural balance and differential dislike. The negative diagonal block is inconsistent with structural balance. We

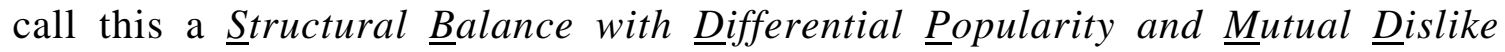
blockmodel and denote it as an SB_DP_MD Model.

To deal with these and other potential complications - including mediation Doreian and Mrvar (2009) proposed 'relaxed structural balance' as a more general model for signed networks. Having only positive blocks and negative blocks was retained. However, they were allowed to appear anywhere in a blockmodel. Relaxed structural balance is a formal generalization of the structural balance. The criterion function, $C_{f}$, as described above and the relocation algorithm were retained for fitting relaxed structural balance models to network data. All that

11 This pattern is present in Figure 2 and this prompted the notion of diagonal negative blocks.

12 One mechanism is disliked attributes of some actors take time to be recognized more widely in a group. 
changed under relaxed structural balance is the potential locations of the signed blocks. Relaxed structural balance permits the statement of another set of ideal blockmodels.

In partial summary, the first two primary substantive hypotheses are stated in a comparative form.

Hypothesis 1 If differential popularity operates for positive ties, there will be a column of positive blocks for the more popular actors and this tendency will increase through time ${ }^{13}$. If structural balance dominates differential popularity then there will be no positive off-diagonal blocks in a column corresponding to universally popular actors. Nor would there be positive off-diagonal blocks for other popular actors.

Hypothesis 2 If differential dislike is operative, there will be a column of negative blocks for the more disliked actors and this tendency will increase though time. In particular, there will be at least one diagonal negative block. If structural balance dominates then there will be no diagonal negative blocks.

Heider's theory is essentially dynamic with actors striving to reduce inconsistencies. This is expressed as a tendency towards balance over time. Indeed, data for examining Heider's theory must be temporal. However, all Heider's imbalanced triples can be balanced in three ways. Alas, Heider was silent on how balance is achieved. It requires complex temporal processes in human groups (Hummon and Doreian, 2003). If differential popularity and differential dislike accumulate over time, this suggests:

Hypothesis 3 Increasing tendencies of differential popularity and differential dislike will create greater inequality on the receipt of both positive and negative ties over time.

The idea of moving towards certain structural forms stems from Heider's notion of tendencies towards balance being extended to relaxed structural balance. The concentration of both positive and negative ties (Hypothesis 3 ) could be the result of two social mechanisms. One is an individual level process where attributes making people popular (liked) or unpopular (disliked) are recognized more over time. The other is found in the idea of actors achieving consistency of views of people as driven by balance. Of course, this leaves open the issue of which of these processes are operative or the extent to which they are both operative. The data at our disposal do not permit an exploration of this issue. Even so, relaxed structural balance incorporates additional processes beyond structural balance.

The tests that we propose are facilitated by using the same criterion function for all fitted models. Relaxed structural balance models have structural balance as a special case. If structural balance dominates all other processes then the SB

13 We allow less extreme versions with some actors more popular but not universally popular as shown in the one variant of the SB_DP Model. Positive valued actor attributes may also take time to be perceived widely. 
Model will be identified implying structural balance is the generating process. But if both structural balance and differential popularity are operating without mutual dislike then a variant of the SB_DP Model will fit the signed data better. And if there is also mutual dislike in subgroups, the SB_DP_MD Model will fit. If any of the more general models within relaxed structural balance fit, there is evidence against structural balance being the sole, or even the main, generating process. Classic structural balance and relaxed structural balance partitions are rivals to be evaluated comparatively. They can be compared through their blockmodel signatures.

\section{Data and methods}

Brusco et al. (2011), based on Leik and Meeker (1975), argue it is more fruitful to have substance, data, and model (with the methods it implies) form a coherent whole. We achieve this here within the rubric of balance theoretic ideas. The SB Model and relaxed structural balance (RSB) models can be evaluated comparatively. Group trajectories towards balance, if they exist, need not imply strictly monotonic change in the level of imbalance. But there will be some overall movement in this direction over time. Given this empirical claim of Heider, it is necessary to examine signed structures over time using blockmodel structures. Given substance drove the hypotheses and the methods of relaxed structural balance are fully consistent with this, the coherence of Leik and Meeker's substance-method-data triple is preserved.

Alas, there are few signed networks over enough time points to test Heider's theory. We know of only two such data sets. One is Newcomb's (1961) data as recorded by Nordlie (1957). The other comes from Sampson's (1968) study of trainee monks in a monastery. Neither data set is ideal. Newcomb collected network data from 17 students in a pseudo-fraternity. In partial exchange for room and board, these previously unacquainted students provided sociometric data for 15 time points over a semester. The strength of Newcomb's study is the network formation process started from an initial state of no network ties. The recorded data were in the form of ranks with each actor ranking all of the other actors in terms of liking. Doreian et al. (1996) recoded these recorded ranked sociometric ties into a signed form. With this recoding, they established reciprocity, transitivity and structural balance had different time scales. The top four ranks were converted to +1 and the bottom three ranks were recoded to -1 . The remaining ties were recoded as zero $^{14}$. We use their (four positive ties and three negative ties) coding scheme here. Of course, as noted by Hallinan (1984) drawing on the arguments of Holland

14 Their reasons for this coding and the formal methods for establishing it are found in their article. With regard to structural balance, other recoding options in terms of the number of positive and negative ties were tried without leading to substantively different results. 
and Leinhardt (1973), there are problems with fixed choice designs. However, as we want our results to be comparable with prior analyses of the Newcomb data we used this coding.

Doreian et al. (1996) computed the imbalance over time for the recoded Newcomb data and showed a general decline over time. While this decline was not strictly monotonic, there was enough support for Heider's empirical hypothesis ${ }^{15}$. However, if the relaxed structural balance model is a better model, one that allows for multiple processes, then imbalance for relaxed structural balance will decline over time. More importantly, imbalance will be lower at each time point than for structural balance. To examine Hypothesis 3, we use Theil's (1967: 92) entropy index, as a measure of inequality, for receiving positive and negative ties at each time point.

The criterion function $C_{f}$ can be viewed as merely descriptive and lacking tests of its utility for partitions established when using it. To address this, we use quadratic assignment regression, QAP, as formulated by Dekker et al. (2007) and implemented in Borgatti et al. (2002), to make statistical assessments of established signed blockmodels. The ideal blockmodels specify (by locations) the presence of positive and negative blocks. Given an established blockmodel (with inconsistencies), we can define the 'fitted' blockmodel that corresponds to the empirical blockmodel. In the following panel we show, on the left, a hypothetical pair of positive and negative blocks with some (bolded) inconsistencies. The c orresponding pair of 'predictions' implied by the blocks in an ideal blockmodel ${ }^{16}$ are on the right.

A positive block (with inconsistencies) The corresponding fitted positive block

$\begin{array}{rrrrrrrr}0 & -1 & 1 & 1 & 0 & 0 & -1 & 0 \\ 1 & 1 & 0 & 1 & -1 & 0 & 1 & -1 \\ 0 & 0 & 1 & 1 & 0 & 0 & 1 & 1 \\ 1 & 0 & 0 & 0 & 1 & 1 & 0 & 1 \\ 1 & 0 & -1 & 0 & 0 & 0 & 0 & -1\end{array}$

$\begin{array}{llllllll}0 & 1 & 1 & 1 & 0 & 0 & 1 & 0 \\ 1 & 1 & 0 & 1 & 1 & 0 & 1 & 1 \\ 0 & 0 & 1 & 1 & 0 & 0 & 1 & 1 \\ 1 & 0 & 0 & 0 & 1 & 1 & 0 & 1 \\ 1 & 0 & 1 & 0 & 0 & 0 & 0 & 1\end{array}$

\footnotetext{
${ }^{15}$ We emphasize the term 'enough support'. In a follow-up study using the Newcomb data, Doreian and Krackhardt (2001) showed that the incidence of two of the imbalanced triples increased over time while the number of two of the balanced triples declined over time.

${ }^{16}$ Borgatti and Everett (1999) propose using Pearsonian correlations in a similar fashion but with a crucial difference. Their ideal blocks are either complete or null. The latter are unproblematic but we differ here by 'predicting' only the implied value of a tie when there is an empirical tie in the data.
} 
A negative block (with inconsistencies)

The corresponding fitted negative block

$\begin{array}{rrrrrrrr}-1 & 0 & 1 & -1 & 0 & 0 & 0 & -1 \\ 1 & -1 & 0 & 0 & 0 & -1 & -1 & 1 \\ -1 & 0 & -1 & 0 & 0 & 0 & -1 & -1 \\ 0 & 0 & -1 & 1 & 1 & -1 & -1 & 0 \\ -1 & 0 & 1 & 0 & 0 & -1 & 0 & 0\end{array}$

$\begin{array}{rrrrrrrr}-1 & 0 & -1 & -1 & 0 & 0 & 0 & -1 \\ -1 & -1 & 0 & 0 & 0 & -1 & -1 & -1 \\ -1 & 0 & -1 & 0 & 0 & 0 & -1 & -1 \\ 0 & 0 & -1 & -1 & -1 & -1 & -1 & 0 \\ -1 & 0 & -1 & 0 & 0 & -1 & 0 & 0\end{array}$

An empirical network with blocks and the fitted blockmodel can be compared by using QAP to assess the fit. QAP is used to 'compare' two whole matrix arrays to examine the extent to which they are the same or consistent with each other. In these analyses, the fitted blockmodel is used to predict the empirical data. If the correlations between the two are significant, the fitted blockmodel passes a test in terms of empirical adequacy. However, if the fit is poor, the blockmodel fails. It is possible also to compare the fitted blockmodel with a random partition as a secondary way of assessing the adequacy of its fit. We did this using the Adjusted Rand Index (ARI) and evaluative criteria put forth by Steinley (2004). He argues ARI values above 0.9 indicate an excellent correspondence in the composition of a pair of partitions; values above 0.8 suggest an acceptable correspondence and values below 0.8 are unacceptable.

Another potential problem with blockmodeling is finding multiple optimal partitions for a given value of $k$. If all have the same block structure, and attention is focused solely on the block structure, this is not a huge problem. But, if there are multiple 'best' partitions, having different block structures, this is a serious problem. A third potential problem is the presence of null blocks: they must be identified. For structural equivalence, only two block types are possible: complete blocks and null blocks. Differential penalties can be imposed on the two types of inconsistencies (ones in null blocks and null ties in complete blocks). Doreian et al. (2004), for partitioning two-mode data, imposed a heavy penalty on the former inconsistency to ensure null blocks appeared as fully null blocks ${ }^{17}$.

For the Newcomb data, there are null blocks. Specifying a null block helps eliminate multiple equally well fitting partitions under relaxed balance. We used the algorithm of Doreian and Mrvar (2009) as implemented in pajek (Batagelj and Mrvar, 1998) for each time point in an inductive fashion with one null block specified. Having identified the 'best' partition structures for $k=4$ inductively, we then, for each time point, pre-specified its delineated block structure in a deductive

17 They used pre-specification but here only the presence of a null block was allowed. 
fashion (with many repetitions) to make sure there were no additional partitions with the identified partition structure ${ }^{18}$.

When comparing relaxed structural balance with structural balance we thought differential popularity would be important and, perhaps, dominate structural balance. The comparisons had to be fair. A crucial difference exists in the behavior of $C_{f}$ as the number of clusters $(k)$ increases fot structural balance and relaxed structural balance. For the former, the curve of the criterion function, $C_{f}$, when plotted against $k$, has a $\mathrm{U}$-shape with a guaranteed minimum value (Doreian et al., 2005: Theorem 10.6). In contrast, for relaxed structural balance, $C_{f}$ decreases monotonically with $k$ (Doreian and Mrvar, 2009: Theorem 4). We chose $k=4$ primarily because the 'best' structural balance results were for this value of $k$. Increasing the value of $k$ beyond 4 has two implications: i) values of $C_{f}$ increase for structural balance while they decrease for relaxed structural balance. This creates a bias favoring the latter for higher values of $k$. For a fair comparative test we used the same value of $k$ for relaxed structural balance and structural balance. If anything, this favored structural balance. At most time points, the optimal partition for structural balance occurs for $k=4$ in the Newcomb data. For the Sampson data, it is $k=3$ at all three time points. We then compared the fitted models with each other ${ }^{19}$.

\section{Empirical results}

\subsection{Using the Newcomb data}

Figure 1 shows the criterion function values for $k=4$ over time for structural and relaxed balance. Both trajectories decrease overall. The values of the criterion function for relaxed balance are always lower than for structural balance, implying the RSB model fits the data better than the SB model. While this has little surprise value, it emphasizes limitations to structural balance. For each time point, we computed the ARI for pairs of partitions obtained from the two models. They ranged from 0.073 to 0.689 . For each time point, the partitions obtained from the two approaches are not the same. Most often, they are not even close.

18 In fitting blockmodels to signed networks where null blocks are specified, the criterion function $C_{f}=\alpha \mathcal{N}+(1-\alpha) D$ was modified by including a term for the null block that ensured that the null block would be as large as possible. (Small null blocks were penalized relative to larger null blocks so larger null blocks were identified.)

19 For Sampson data we consider also $k=4$ for relaxed structural balance. 


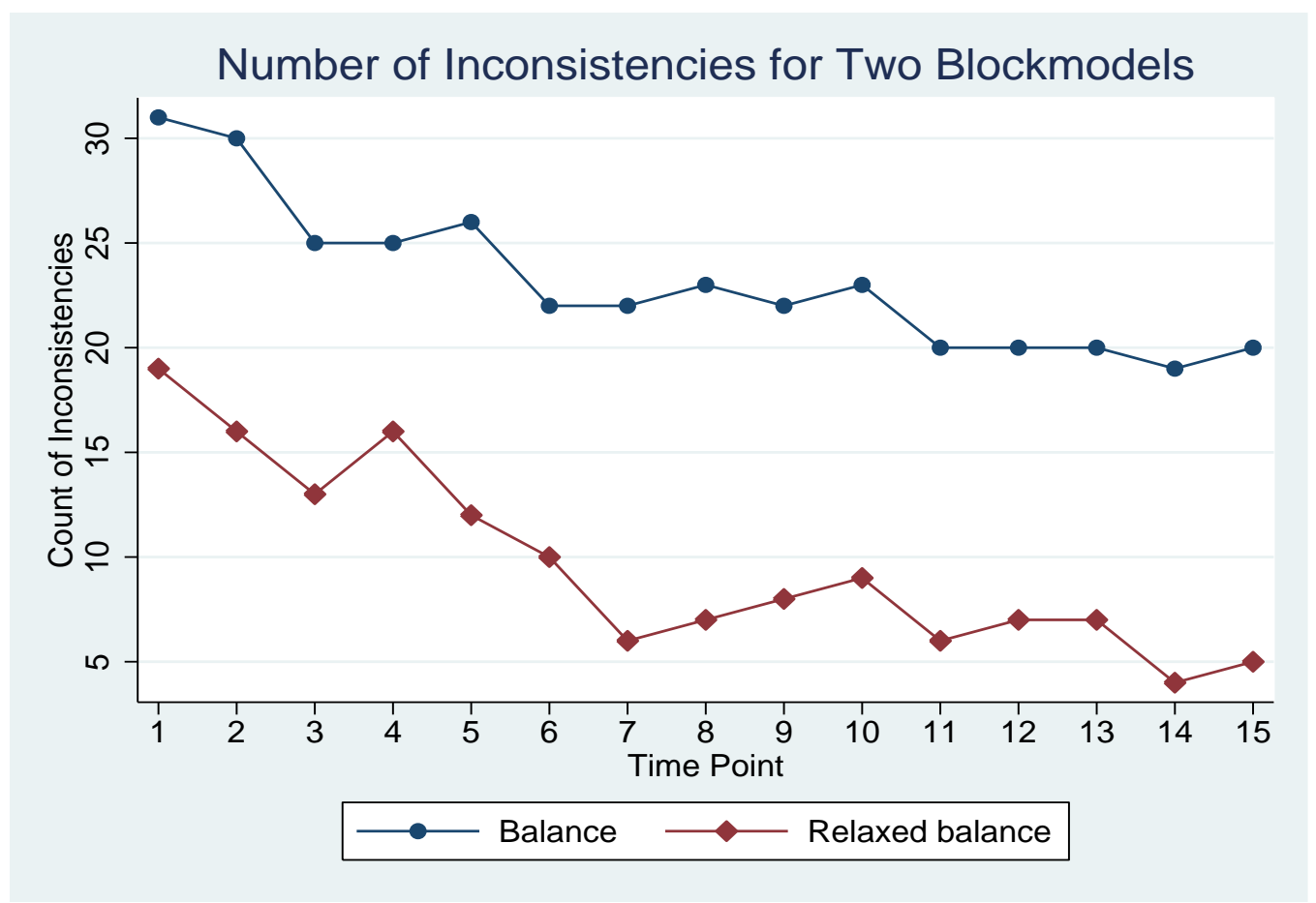

Figure 1: Inconsistency counts for the Structural Balance and the Relaxed Structural Balance models: Newcomb data.

There are additional issues in fitting blockmodels to network data meriting attention. The first concerns the predictive value of the fitted blockmodels. We computed the correlation, for the 15 time points labeled $t_{1}$ through $t_{15}$, between these QAP correlations and the value of the criterion function, $\mathrm{C}_{\mathrm{f}}$. The value of this correlation is $-0.959(\mathrm{p}<.0001)$ indicating an very close correspondence between the two set of values. Table 1 provides the numerical values and the QAP correlations for both relaxed structural balance and structural balance. The QAP correlations in Table 1, using a permutation test, act as a close proxy for a permutation test for the criterion function. The $\mathrm{p}$-values ${ }^{20}$ for the QAP correlations are all less than 0.001. The values for structural balance have a similar temporal pattern but the correlation between the QAP correlations and the criterion function is slightly less. Even so, the lower QAP correlations for structural balance suggest poorer predictive performances consistent with the values of the criterion function for the two rival models.

20 Most correlations are 'significant' which may be an inherent feature of QAP. However, our use of QAP is driven primarily by a need to compare the results from using relaxed structural balance and structural balance. It is unlikely that a bias towards significance affects the comparative results differently. Also there are non-significant QAP estimates in the results we report. 
Table 1: QAP correlations and criterion function values: empirical and fitted blockmodels: Newcomb data.

\begin{tabular}{|l|c|c|c|c|}
\hline$T$ & $\begin{array}{c}\text { RSB QAP } \\
\text { Correlations* }\end{array}$ & $\begin{array}{c}\text { RSB Criterion Function } \\
\text { Values }\left(\mathrm{C}_{\mathrm{f}}\right)\end{array}$ & $\begin{array}{c}\text { SB QAP } \\
\text { Correlations* }\end{array}$ & $\begin{array}{c}\text { SB Criterion Function } \\
\text { Values }\left(\mathrm{C}_{\mathrm{f}}\right)\end{array}$ \\
\hline $\mathrm{t}_{1}$ & 0.679 & 9.5 & 0.499 & 15.5 \\
\hline $\mathrm{t}_{2}$ & 0.740 & 8.0 & 0.502 & 15.0 \\
\hline $\mathrm{t}_{3}$ & 0.779 & 6.5 & 0.588 & 12.5 \\
\hline $\mathrm{t}_{4}$ & 0.752 & 8.0 & 0.598 & 12.5 \\
\hline $\mathrm{t}_{5}$ & 0.810 & 6.0 & 0.579 & 13.0 \\
\hline $\mathrm{t}_{6}$ & 0.754 & 5.0 & 0.511 & 11.0 \\
\hline $\mathrm{t}_{7}$ & 0.911 & 3.0 & 0.633 & 11.0 \\
\hline $\mathrm{t}_{8}$ & 0.881 & 3.5 & 0.619 & 11.5 \\
\hline $\mathrm{t}_{9}$ & 0.865 & 4.0 & 0.633 & 11.0 \\
\hline $\mathrm{t}_{10}$ & 0.860 & 4.5 & 0.617 & 11.5 \\
\hline $\mathrm{t}_{11}$ & 0.899 & 3.0 & 0.674 & 10.0 \\
\hline $\mathrm{t}_{12}$ & 0.898 & 3.0 & 0.669 & 10.0 \\
\hline $\mathrm{t}_{13}$ & 0.881 & 3.5 & 0.671 & 10.0 \\
\hline $\mathrm{t}_{14}$ & 0.932 & 2.0 & 0.687 & 9.5 \\
\hline $\mathrm{t}_{15}$ & 0.915 & 2.5 & 0.669 & 10.0 \\
\hline
\end{tabular}

RSB Relaxed Structural Balance; SB Structural Balance

* All p-values $<0.001$. The correlation between QAP correlations and $\mathrm{C}_{\mathrm{f}}$ is -0.959 for RSB and 0.858 for SB.

Table 2 presents the results of using QAP regressions comparing the predictive values of RSB and SB. Reading from the right, it appears both the fitted SB and the fitted RSB blockmodels have some predictive value. Further, the predictive value for each, roughly, increases through time. However, when the fitted SB blockmodel is included as a predictor with the fitted RSB blockmodel it seldom increases the predictive value of the QAP regression. Of course, when two predictors are correlated there is no unique partition of the variance explained between them. However, we note the following additional items in Table 2: i) the estimated intercept is near zero for each time point; ii) the unstandardized coefficients are such that the coefficients for RSB are always larger than the corresponding coefficients for $\mathrm{SB}^{21}$; iii) over time, the unstandardized coefficient for SB declines while the unstandardized coefficients for RSB increase; and iv) at each time point, the standardized coefficient for RSB is larger than the standardized coefficient for SB indicating it as the more potent predictor. In short, the fitted RSB blockmodel has superior predictive value than the fitted SB blockmodel.

${ }^{21}$ The two fitted blockmodels have the same density so there is not an issue of different scales inflating one coefficient relative to the other. 
Table 2: QAP Regressions comparing Relaxed Structural Balance and Structural Balance: Newcomb data.

\begin{tabular}{|c|c|c|c|c|c|c|c|}
\hline $\mathrm{T}$. & Variable & $\begin{array}{c}\text { Unstandardiz } \\
\text { Coefficient }\end{array}$ & $\begin{array}{c}\text { d Standardized } \\
\text { Coefficient }\end{array}$ & $\mathrm{p}$-value & $\mathrm{R}^{2}$ & $\begin{array}{c}\mathrm{R}^{2} \\
\text { (for } \mathrm{RSB} \text { ) }\end{array}$ & $\begin{array}{c}\mathrm{R}^{2} \\
\text { (for } \mathrm{SB} \text { ) }\end{array}$ \\
\hline $\mathrm{t}_{1}$ & $\begin{array}{l}\text { Intercept } \\
\text { SB } \\
\text { RSB }\end{array}$ & $\begin{array}{l}0.051 \\
0.134 \\
0.596\end{array}$ & $\begin{array}{l}0.000 \\
0.133 \\
0.598\end{array}$ & $\begin{array}{l}-\overline{0} \\
0.0140 \\
0.0005\end{array}$ & 0.47 & 0.46 & 0.25 \\
\hline $\mathrm{t}_{2}$ & $\begin{array}{l}\text { Intercept } \\
\text { SB } \\
\text { RSB }\end{array}$ & $\begin{array}{l}0.015 \\
0.201 \\
0.651\end{array}$ & $\begin{array}{l}0.000 \\
0.202 \\
0.646\end{array}$ & $\begin{array}{l}- \\
0.0005 \\
0.0005\end{array}$ & 0.58 & 0.55 & 0.25 \\
\hline $\mathrm{t}_{3}$ & $\begin{array}{l}\text { Intercept } \\
\text { SB } \\
\text { RSB }\end{array}$ & $\begin{array}{l}0.013 \\
0.208 \\
0.662\end{array}$ & $\begin{array}{l}0.000 \\
0.209 \\
0.659\end{array}$ & $\begin{array}{l}-\bar{l} \\
0.0005 \\
0.0005\end{array}$ & 0.64 & 0.61 & 0.35 \\
\hline $\mathrm{t}_{4}$ & $\begin{array}{l}\text { Intercept } \\
\text { SB } \\
\text { RSB }\end{array}$ & $\begin{array}{l}0.042 \\
0.285 \\
0.610\end{array}$ & $\begin{array}{l}0.000 \\
0.284 \\
0.604\end{array}$ & $\begin{array}{l}-\bar{l} \\
0.0005 \\
0.0005\end{array}$ & 0.62 & 0.57 & 0.36 \\
\hline $\mathrm{t}_{5}$ & $\begin{array}{l}\text { Intercept } \\
\text { SB } \\
\text { RSB }\end{array}$ & $\begin{array}{l}0.041 \\
0.089 \\
0.753\end{array}$ & $\begin{array}{l}0.000 \\
0.089 \\
0.752\end{array}$ & $\begin{array}{l}- \\
0.0265 \\
0.0005\end{array}$ & 0.66 & 0.66 & 0.34 \\
\hline $\mathrm{t}_{6}$ & $\begin{array}{l}\text { Intercept } \\
\text { SB } \\
\text { RSB }\end{array}$ & $\begin{array}{l}0.010 \\
0.085 \\
0.704\end{array}$ & $\begin{array}{l}0.000 \\
0.085 \\
0.702\end{array}$ & $\begin{array}{l}- \\
0.0365 \\
0.0005\end{array}$ & 0.57 & 0.57 & 0.26 \\
\hline $\mathrm{t}_{7}$ & $\begin{array}{l}\text { Intercept } \\
\text { SB } \\
\text { RSB }\end{array}$ & $\begin{array}{r}-0.008 \\
0.076 \\
0.868\end{array}$ & $\begin{array}{l}0.000 \\
0.077 \\
0.861\end{array}$ & $\begin{array}{l}-\overline{0} \\
0.0100 \\
0.0005\end{array}$ & 0.83 & 0.83 & 0.40 \\
\hline $\mathrm{t}_{8}$ & $\begin{array}{l}\text { Intercept } \\
\text { SB } \\
\text { RSB }\end{array}$ & $\begin{array}{l}0.004 \\
0.051 \\
0.848\end{array}$ & $\begin{array}{l}0.000 \\
0.051 \\
0.847\end{array}$ & $\begin{array}{l}- \\
0.0880 \\
0.0005\end{array}$ & 0.78 & 0.78 & 0.38 \\
\hline$t_{9}$ & $\begin{array}{l}\text { Intercept } \\
\text { SB } \\
\text { RSB }\end{array}$ & $\begin{array}{r}-0.020 \\
0.172 \\
0.767\end{array}$ & $\begin{array}{l}0.000 \\
0.173 \\
0.761\end{array}$ & $\begin{array}{l}- \\
0.0005 \\
0.0005\end{array}$ & 0.77 & 0.75 & 0.40 \\
\hline $\mathrm{t}_{10}$ & $\begin{array}{l}\text { Intercept } \\
\text { SB } \\
\text { RSB }\end{array}$ & $\begin{array}{l}0.028 \\
0.108 \\
0.792\end{array}$ & $\begin{array}{l}0.000 \\
0.108 \\
0.791\end{array}$ & $\begin{array}{l}- \\
0.0040 \\
0.0005\end{array}$ & 0.75 & 0.74 & 0.38 \\
\hline $\mathrm{t}_{11}$ & $\begin{array}{l}\text { Intercept } \\
\text { SB } \\
\text { RSB }\end{array}$ & $\begin{array}{l}0.021 \\
0.022 \\
0.881\end{array}$ & $\begin{array}{l}0.000 \\
0.022 \\
0.883\end{array}$ & $\begin{array}{l}-\bar{l} \\
0.2289 \\
0.0005\end{array}$ & 0.81 & 0.81 & 0.45 \\
\hline $\mathrm{t}_{12}$ & $\begin{array}{l}\text { Intercept } \\
\text { SB } \\
\text { RSB }\end{array}$ & $\begin{array}{r}-0.026 \\
-0.069 \\
0.957 \\
\end{array}$ & $\begin{array}{r}0.000 \\
-0.069 \\
0.952\end{array}$ & $\begin{array}{l}- \\
0.0475 \\
0.0005\end{array}$ & 0.81 & 0.81 & 0.45 \\
\hline $\mathrm{t}_{13}$ & $\begin{array}{l}\text { Intercept } \\
\text { SB } \\
\text { RSB }\end{array}$ & $\begin{array}{ll}\text { t } & 0.006 \\
0.071 \\
0.831 \\
\end{array}$ & $\begin{array}{l}0.000 \\
0.071 \\
0.830\end{array}$ & $\begin{array}{l}- \\
0.0440 \\
0.0005\end{array}$ & 0.78 & 0.78 & 0.45 \\
\hline $\mathrm{t}_{14}$ & $\begin{array}{l}\text { Intercept } \\
\text { SB } \\
\text { RSB }\end{array}$ & $\begin{array}{r}-0.004 \\
0.084 \\
0.876\end{array}$ & $\begin{array}{l}0.000 \\
0.085 \\
0.874\end{array}$ & $\begin{array}{l}-\bar{l} \\
0.0060 \\
0.0005\end{array}$ & 0.87 & 0.87 & 0.47 \\
\hline $\mathrm{t}_{15}$ & $\begin{array}{l}\text { Intercept } \\
\text { SB } \\
\text { RSB }\end{array}$ & $\begin{array}{r}-0.000 \\
0.020 \\
0.902\end{array}$ & $\begin{array}{l}0.000 \\
0.020 \\
0.901\end{array}$ & $\begin{array}{l}-\overline{0} \\
0.1964 \\
0.0005\end{array}$ & 0.84 & 0.84 & 0.45 \\
\hline
\end{tabular}


The blockmodels for each time point are in Table 3 in three panels. The first row in each box gives the specific time point. The second row shows whether the partition reported was unique. A unique partition for 13 of the 15 time points was returned. For one time point $\left(\mathrm{t}_{8}\right)$ there are two partitions. In each case, the block structure is the same and the partitions differ only by a 'floater' moving between a pair of clusters $^{22}$. For $t_{12}$, there were multiple partitions but one stands out ${ }^{23}$. The third row gives the value of the criterion function for $\alpha=0.5$ (the inconsistency count is double the criterion function values reported in Figure 1). The final row in each cell gives the block structure where $\mathrm{P}, \mathrm{N}$ and $\mathrm{O}$ denote, respectively, positive, negative and null blocks.

Table 3: Signed block structures over 15 time points: Newcomb data*.

\begin{tabular}{|c|c|c|c|c|}
\hline$t_{1}$ & $t_{2}$ & $t_{3}$ & $t_{4}$ & $t_{5}$ \\
\hline Unique & Unique & Unique & Unique & Unique \\
\hline$X(P)=9.5$ & $X(P)=8.0$ & $X(P)=6.5$ & $X(P)=8.0$ & $X(P)=6.0$ \\
\hline PPNN & PNPN & PNPN & POPN & PNPN \\
\hline PONN & PPNN & PPNN & PPNP & PPNN \\
\hline PNPN & PNNP & PNNP & PNPN & PPNN \\
\hline PNNP & NPPO & NPPO & PPNN & PNON \\
\hline$t_{6}$ & $t_{7}$ & $t_{8}$ & t9 & $t_{10}$ \\
\hline Unique & Unique & Two & Unique & Unique \\
\hline$X(P)=5$ & $X(P)=3.0$ & $X(P)=3.5$ & $X(P)=4.0$ & $X(P)=4.5$ \\
\hline PPON & POPN & PPPN & PPPN & PNPN \\
\hline PNPN & PPNN & PNPN & PPNN & OPPN \\
\hline PPPN & PPPN & PPON & PNON & PPNN \\
\hline PPNN & PPNN & PPNN & PPPN & PNNN \\
\hline$t_{11}$ & $t_{12}$ & $t_{13}$ & $t_{14}$ & $t_{15}$ \\
\hline Unique & Unique** & Unique & Unique & Unique \\
\hline$X(P)=3.0$ & $X(P)=3.0$ & $X(P)=3.5$ & $X(P)=2.0$ & $X(P)=2.5$ \\
\hline PPPN & PPNN & PPNN & PPNN & PPPN \\
\hline PPON & PPNN & POPN & PNNN & PPON \\
\hline PNPN & PNOP & PPNN & PONN & PNPN \\
\hline PPNN & PNPN & PNNN & PNPN & PNNN \\
\hline
\end{tabular}

*P denotes a positive block, $\mathrm{N}$ denotes a negative block and $\mathrm{O}$ denotes a null block.

** See footnote 15 for an explanation of this.

\footnotetext{
22 The value of the ARI measure is 0.845 which is in the acceptable range specified by Steinley (2004).

${ }^{23}$ For $t_{12}$, it was necessary to specify two null blocks to have a unique solution. One of the identified null blocks contained a negative tie. We treated it (the third block in the first row) as a negative block. While there were multiple partitions using one specified null block, one is shown in Table 1. Specifying a second null block suggests a way of choosing a partition from the multiple equally well fitting partitions.
} 
We examined the delineated signed blockmodel at each time point. We note that, especially towards the end of the process, the composition of the positions in terms of membership is quite stable. There are members of positions remaining firmly in place while a few do move between positions in transitions. We note also that the sizes of positions do not change abruptly in each transition. Illustrating the different partitions for structural balance and relaxed structural balance we show their unique partitions at $\mathrm{t}_{14}$ for $k=4$ in Figure 2 . We chose this time point because it is near the end of the network evolution and the criterion functions are lowest at $\mathrm{t}_{14}$ for both models: each structure is closest to its ideal structure. The black squares represent positive ties with negative ties represented by grey diamonds. The SB partition is in the top panel. The RSB partition is in the bottom panel. The number of inconsistencies for structural balance is 19 while the corresponding number is 4 for relaxed balance. The reason for the sharp drop in the number of inconsistencies is clear. Structural balance struggles with the large number of off-diagonal positive ties. Also, the structural balance partition is unsatisfactory because it returns a partition with one large cluster, one pair, and two singletons. It misses the mutually hostile subgroup completely because negative blocks cannot appear on the main diagonal. The RSB partition returns an optimal partition with clusters of size 9, 3, 3 and 2. Many of the positive off-diagonal blocks are part of a coherent structure instead of contributing inconsistencies under structural balance. In short, the SB_DP_MD model fits these $\left(\mathrm{t}_{14}\right)$ data far better than the SB model.

It is apparent from Table 3 that none of the fitted RSB blockmodels conform to the SB Model. From Figure 1, the SB Model fares less well than a relaxed structural balance model, consistent with results shown in Table 2. Structural balance cannot be viewed as the sole generating process for these data. It may not be the dominant process. We next interpret the results in Table 3.

Differential popularity and Hypothesis 1 are considered first. The top left block is positive for all time points, a result consistent with both structural balance and differential popularity. The column of positive blocks in the left hand column is present for 12 of the 15 time points, including the last 5 leading to the final evolved structure. For $t_{2}$ and $t_{3}$, a negative off-diagonal block appears in this column. Even so, there are still two positive off-diagonal blocks. There is one null block with two positive blocks in the first column at $\mathrm{t}_{10}$. This pattern provides overwhelming support for the presence of differential popularity (Hypothesis 1) and overwhelming support for Feld and Elsmore's (1984) arguments for it as a generative process. Hypothesis 1 is resolved in favor of the SB_DP model. A column of positive blocks appears early and is present for most time points. This feature is stable with decreasing inconsistencies. 


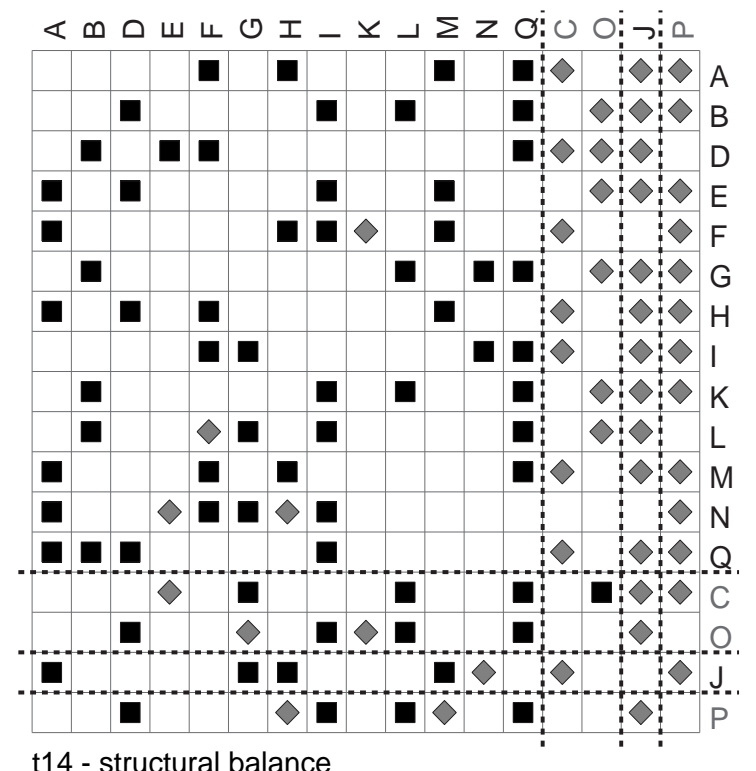

t14 - structural balance

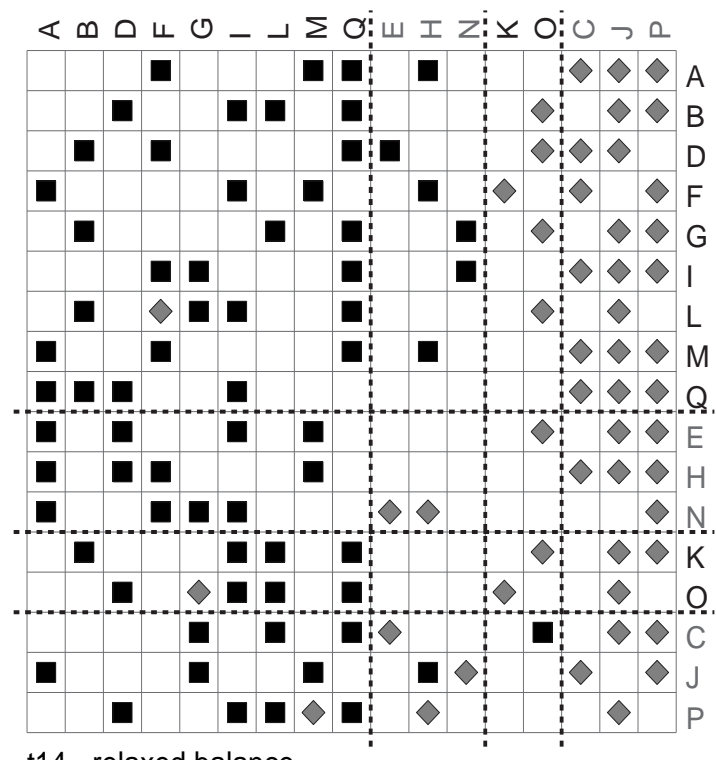

t14 - relaxed balance

Figure 2: Structural Balance and Relaxed Balance partitions at t14 (Newcomb data).

Next, we consider Hypothesis 2. For differential dislike, including mutual dislike, the column of negative blocks on the right first appears at $t_{5}$. It was not there at the outset and emerged over time. It persisted through all subsequent time points. The bottom right (diagonal) negative block reveals a subgroup with mutual dislike. This also contradicts structural balance. However, negative offdiagonal blocks in this column are consistent with structural balance and differential dislike. Features of the SB_DP_MD Model are evident at multiple time points. Hypothesis 2 is resolved in favor of the SB_DP_MD model. There is evidence of differential popularity emerging earlier with a shorter time scale than differential dislike.

The signs of the blocks in the middle two columns for each of the fitted signed blockmodels have been treated as having secondary interest. Yet, for structural balance theory, additional positive blocks off the main diagonal and negative blocks on it provides further contradictory evidence. For eight time points there is one negative block on the main diagonal and for six there are two such negative blocks. There is strong evidence for differential popularity - in both a universal and less universal sense - as well as mutual dislike within a set of actors. These features are disentangled from balance processes because they leave observable traces inconsistent with that theory. Consistent with Hallinan's (1984) observation, structural features suggest the operation of multiple processes. Some cannot be completely distinguished by looking solely at blocks. However, there is some further evidence in favor of differential dislike.

The ideas of differential popularity and differential dislike imply that both positive and negative ties are concentrated on some actors but not on others. A 
natural way of considering this is by examining inequality in the receipt of these ties. Our third hypothesis claims that this inequality will increase over time. Figure 3 shows the values of the Theil entropy index over time ${ }^{24}$. Very similar results hold when the coefficient of variation (standard deviation/mean) or the Gini coefficient is used. The inequality for receiving negative ties increases over the first 7 time points, shows some oscillation for the next three time points, followed by a downwards drift, and then some more oscillation with increasing values. The over-time movement of inequality for the receipt of positive ties is quite different. It is flat over the first four time points, increases from $t_{4}$ through $t_{7}$, drops, and then oscillates while increasing. The inequality in the receipt of negative ties is always much higher than for the receipt of positive ties after $t_{1}$. The third hypothesis is strongly supported for received negative ties while, at best, it is supported for the receipt of positive ties from $t_{4}$ through $t_{7}$ and only weakly supported after $t_{7}$. The greater concentration of negative ties over time suggests that differential dislike generates more of the column of negative blocks than structural balance.

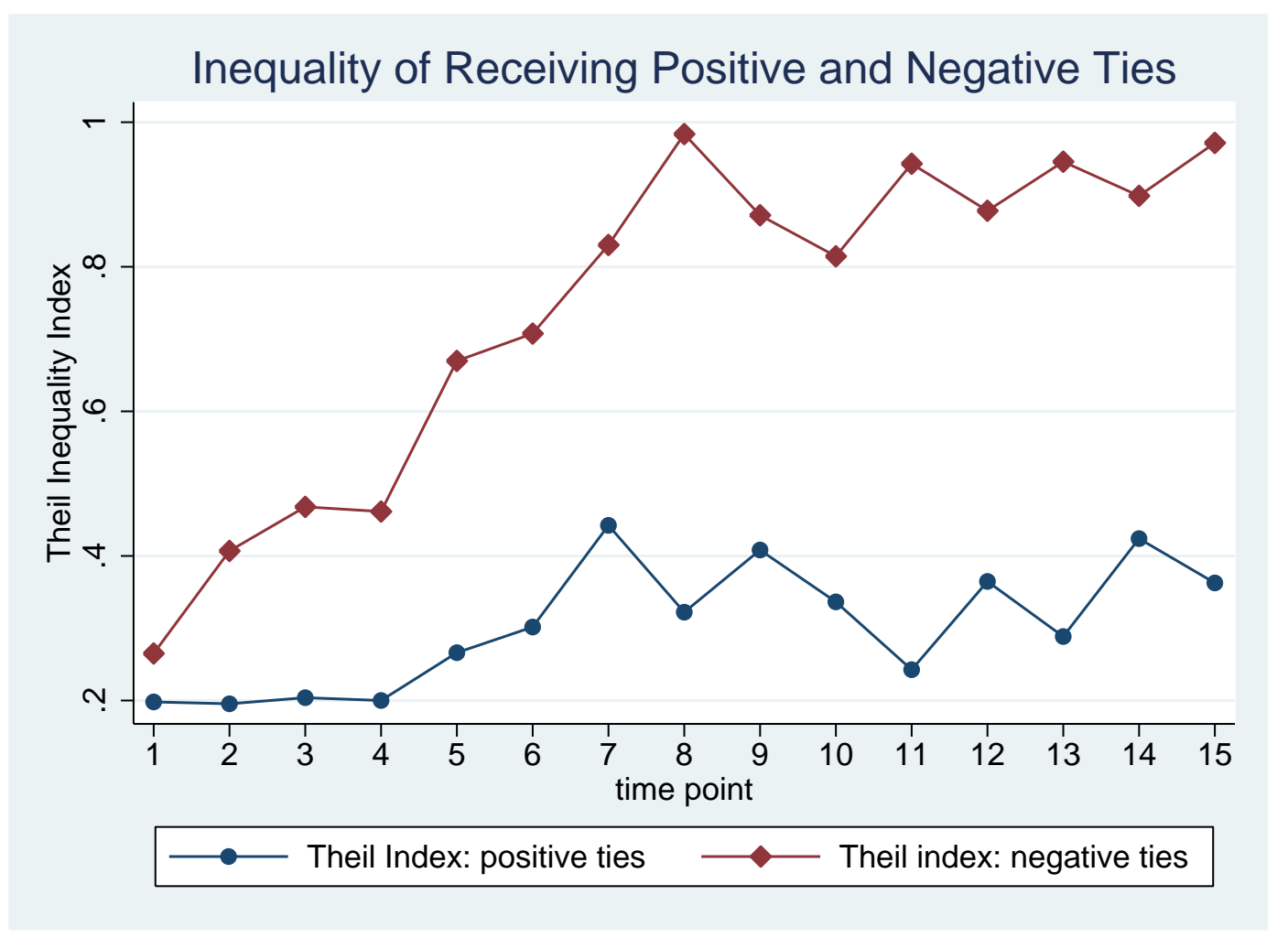

Figure 3: Inequalities in receiving positive and negative ties: Newcomb data.

24 The results in Figure 3 are not due to having 4 positive ties and 3 negative ties from each actor. The trajectory of the Theil index, when using only 3 positive ties, is close to the trajectory of the index for 4 positive ties. 


\subsection{Using the Sampson Data}

Sampson's (1968) data has three time points (labeled in the literature as $\mathrm{T}_{2}, \mathrm{~T}_{3}$, and $\mathrm{T}_{4}$. Sampson collected data for an earlier time point ${ }^{25}\left(\mathrm{~T}_{1}\right)$. He collected signed data on four relations: affect, esteem, influence, and sanction. Each took an apparent metric form with three ranked positive and three ranked negative ties. The sanction relation is problematic because some trainee monks refused to provide data (or claimed they sanctioned no-one). Doreian (2008) argued for using a multi-indicator approach for multiple relations. We do this here. We summed the binarized ${ }^{26}$ affect, esteem and influence relations. The valued signed relation is the number of ties with a specific sign between pairs of actors. From prior analyses (Sampson, 1968; Breiger et al., 1975; Doreian and Mrvar, 1996), we know there are $k=3$ clusters of monks. Figure 4 shows three trajectories for the criterion function. Two are for SB and RSB for $k=3$. We compare these first. The trajectory of the criterion function for relaxed structural balance for $k=4$ has additional interest value regarding differential popularity.

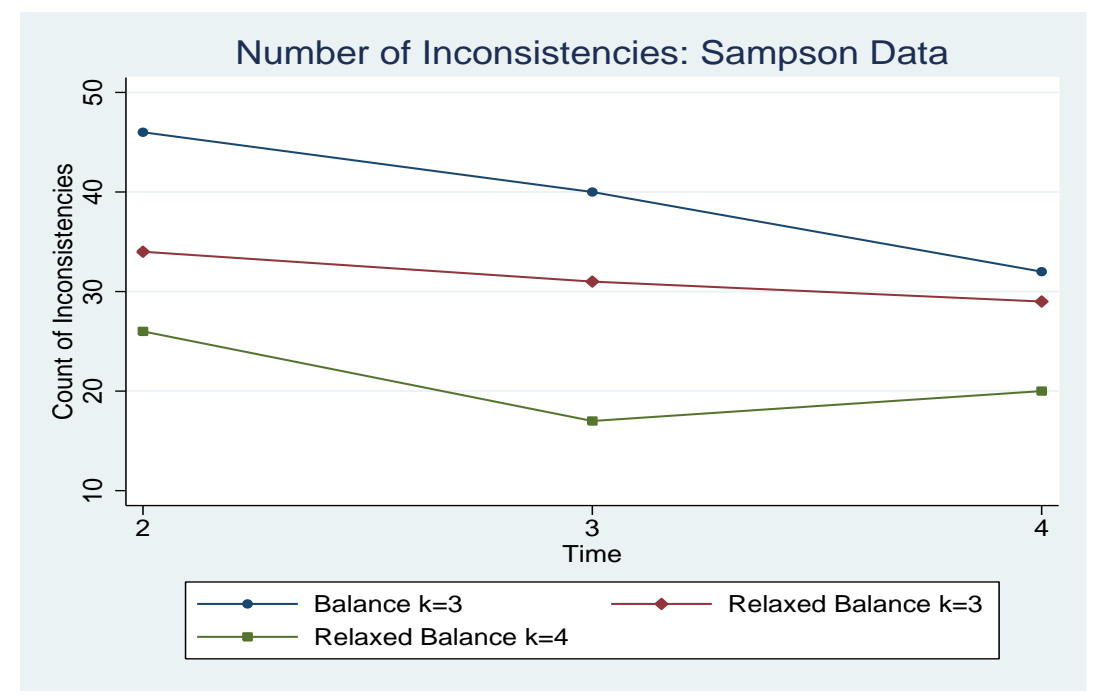

Figure 4: Inconsistency counts for the Structural Balance and the Relaxed Structural Balance models: Sampson data.

25 The $\mathrm{T}_{1}$ data were for a different set of monks. Some of them departed before $\mathrm{T}_{2}$. Those who remained were joined by a group on new trainee monks at $T_{2}$.

26 This was done because summing the ranks seems problematic with regard to measurement. The value of Cronbach's $\alpha$ for the three time points considered here are $0.795\left(\mathrm{~T}_{2}\right), 0.777\left(\mathrm{~T}_{3}\right)$ and 0 . $849\left(\mathrm{~T}_{4}\right)$, suggesting these three network relations are very consistent from a measurement point of view. Also, the comparisons of random partitions of the Sampson data into the same number of positions with the relaxed balance theoretic partitions, that value of the ARI ranges between -0.06 through -0.02 over the partitions reported in Table 3 . 
Both trajectories for $k=3$ decline over time. The values of the criterion function for RSB are smaller than for SB. However, this evidence is modest: the declines for the RSB are small. For the last time point, the two values of the criterion function are close. The value of the criterion function for the RSB model for $k=4$ declines from the first time point to the second but rises slightly at the third time point ${ }^{27}$. The values of the QAP correlations for $k=3$ are: $0.708\left(\mathrm{~T}_{2}\right)$; $0.687\left(\mathrm{~T}_{3}\right)$ and $0.737\left(\mathrm{~T}_{4}\right)$. And for $k=4$ they are: $0.760\left(\mathrm{~T}_{2}\right) ; 0.871\left(\mathrm{~T}_{3}\right)$ and 0.816 $\left(\mathrm{T}_{4}\right)$. For all these QAP correlations $\mathrm{p}<0.001$ confirming the descriptive values for the criterion function, $C_{f}$, are noteworthy.

Table 4: Signed block structures over 3 time points: Sampson data*.

Structural balance $(\mathrm{k}=3)$

\begin{tabular}{|l|l|l|}
\hline $\mathrm{T}_{2}$ & $\mathrm{~T}_{3}$ & $\mathrm{~T}_{4}$ \\
Unique & Unique & Unique \\
$\mathrm{X}(\mathrm{P})=23$ & $\mathrm{X}(\mathrm{P})=20$ & $\mathrm{X}(\mathrm{P})=16$ \\
PNN & PNN & PNN \\
NPN & NPN & NPN \\
NNP & NNP & NNP \\
& & \\
\hline
\end{tabular}

Relaxed balance $(\mathrm{k}=3)$

\begin{tabular}{|l|l|l|}
\hline $\mathrm{T}_{2}$ & $\mathrm{~T}_{3}$ & $\mathrm{~T}_{4}$ \\
Unique & Unique & Unique \\
$\mathrm{X}(\mathrm{P})=17$ & $\mathrm{X}(\mathrm{P})=15.5$ & $\mathrm{X}(\mathrm{P})=14.5$ \\
$\mathrm{PNN}$ & $\mathrm{PNN}$ & $\mathrm{PNN}$ \\
$\mathrm{PPN}$ & $\mathrm{PPN}$ & $\mathrm{PPN}$ \\
$\mathrm{NNP}$ & $\mathrm{NNP}$ & $\mathrm{NNP}$ \\
& & \\
\hline
\end{tabular}

Relaxed balance $(\mathrm{k}=4)$

\begin{tabular}{|l|l|l|}
\hline $\mathrm{T}_{2}$ & $\mathrm{~T}_{3}$ & $\mathrm{~T}_{4}$ \\
Unique & Unique & Unique \\
$\mathrm{X}(\mathrm{P})=13$ & $\mathrm{X}(\mathrm{P})=8.5$ & $\mathrm{X}(\mathrm{P})=10$ \\
$\mathrm{PNNN}$ & $\mathrm{PNPN}$ & $\mathrm{PPNP}$ \\
$\mathrm{PPNN}$ & PPNN & PPNN \\
PNPP & PNPP & PPPN \\
NNPP & NNPP & PNNP \\
& & \\
\hline
\end{tabular}

*P denotes a positive block, $\mathrm{N}$ denotes a negative block, $\mathrm{O}$ denotes a null block

27 One problem with Sampson's data is the small number of time points. Also, the data collection, in contrast to Newcomb's data, did not start from a null network. 
Table 4 presents the corresponding signed blockmodels for the three time points. For $k=3$, there are no large differences between the two blockmodels. The blockmodel for structural balance must be the SB model. For RSB, the same blockmodel existed at each time point with just one difference from the SB model: for all time points, one positive off-diagonal block is in the first column of blocks. In terms of Hypothesis 1, only a modest version of the SB_DP is present at each time point. Even so, it provided slightly better fits. Table 5 reports QAP regressions for the Sampson data. The top panel concerns the $k=3$ partitions. The RSB effect dominates SB only for $\mathrm{T}_{2}$, consistent with the larger difference in the values of the criterion function at this time point in Table 3. In terms of Hypothesis 2, there is no for a SB_DP_MD model given the absence of a negative diagonal block. The off-diagonal negative blocks are consistent with both structural balance and differential dislike.

Table 5: QAP Regressions comparing Relaxed Structural Balance and Structural Balance: Sampson data.

A: Three positions $(\mathrm{k}=3)$

\begin{tabular}{|l|lrrr|r|r|c|}
\hline $\mathrm{T}$ & Variable & $\begin{array}{c}\text { Unstandardized } \\
\text { Coefficient }\end{array}$ & $\begin{array}{c}\text { Standardized p-value } \\
\text { Coefficient }\end{array}$ & $\mathrm{R}^{2}$ & $\mathrm{R}^{2}$ & $\mathrm{R}^{2}$ \\
$(\mathrm{RSB})$ & & & & \\
\hline $\mathrm{T}_{2}$ & Intercept & 0.111 & 0.000 & - & 0.67 & 0.67 & 0.53 \\
& $\mathrm{SB}$ & 0.040 & 0.039 & 0.2324 & & & \\
& $\mathrm{RSB}$ & 0.782 & 0.782 & 0.0005 & & & \\
\hline $\mathrm{T}_{3}$ & Intercept & 0.107 & 0.000 & - & 0.67 & 0.66 & 0.53 \\
& $\mathrm{SB}$ & 0.174 & 0.173 & 0.0075 & & & \\
& $\mathrm{RSB}$ & 0.672 & 0.672 & 0.0005 & & & \\
\hline $\mathrm{T}_{4}$ & Intercept & 0.057 & 0.000 & - & 0.77 & 0.73 & 0.68 \\
& SB & 0.356 & 0.355 & 0.0005 & & & \\
& RSB & 0.556 & 0.556 & 0.0005 & & & \\
\hline
\end{tabular}

RSB Relaxed Structural Balance; SB Structural Balance

B: Four positions $(k=4)$ RSB only

\begin{tabular}{|l|lccc|c|}
\hline Time & Variable & $\begin{array}{c}\text { Unstandardized } \\
\text { Coefficient }\end{array}$ & $\begin{array}{c}\text { Standardized } \\
\text { Coefficient }\end{array}$ & p-value & $\mathrm{R}^{2}$ \\
\hline $\mathrm{T}_{2}$ & Intercept & 0.031 & 0.000 & - & 0.74 \\
& RSB & 0.858 & 0.859 & 0.0005 & - \\
\hline $\mathrm{T}_{3}$ & Intercept & -0.001 & 0.000 & 0.0005 & \\
& RSB & 0.889 & 0.889 & - & 0.81 \\
\hline $\mathrm{T}_{4}$ & Intercept & -0.045 & 0.000 & 0.0005 & \\
& RSB & 0.903 & 0.902 & & \\
\hline
\end{tabular}

The lowest panel of Table 4 displays the blockmodel structure for relaxed balance with $k=4$. The evidence in these blockmodels is stronger for a SB_DP 
model fitting the Sampson data because of the presence of more off-diagonal positive blocks. At the last time point, $\mathrm{T}_{4}$, there is a full column of positive blocks in the RSB blockmodel as well as other off-diagonal positive blocks. While structural balance works well for the Sampson data for $k=3$, for $k=4$ there is stronger evidence in favor of the SB_DP model. The corresponding results for prediction using only the RSB fitted blockmodel for the $k=4$ are provided in the lower panel of Table 5. This fitted blockmodel is a potent predictor of the signed relation for all three time points.

Figure 5 shows the structural balance partitions of the Sampson data for each time point. They are consistent with prior analyses with three clusters of actors: The Young Turks (John Bosco, Gregory, Mark, Winfrid, Hugh, Boniface and Albert); the Loyal Opposition (Peter, Bonaventure, Berthold, Ambrose, Victor, Romauld, Louis and Amand), and the Outcasts (Basil, Elias and Simplicius) were identified by Sampson (1968). There are some minor differences with Ambrose being in the Young Turk cluster at T3 and Amand joining the Outcasts ${ }^{28}$ at $\mathrm{T}_{4}$.
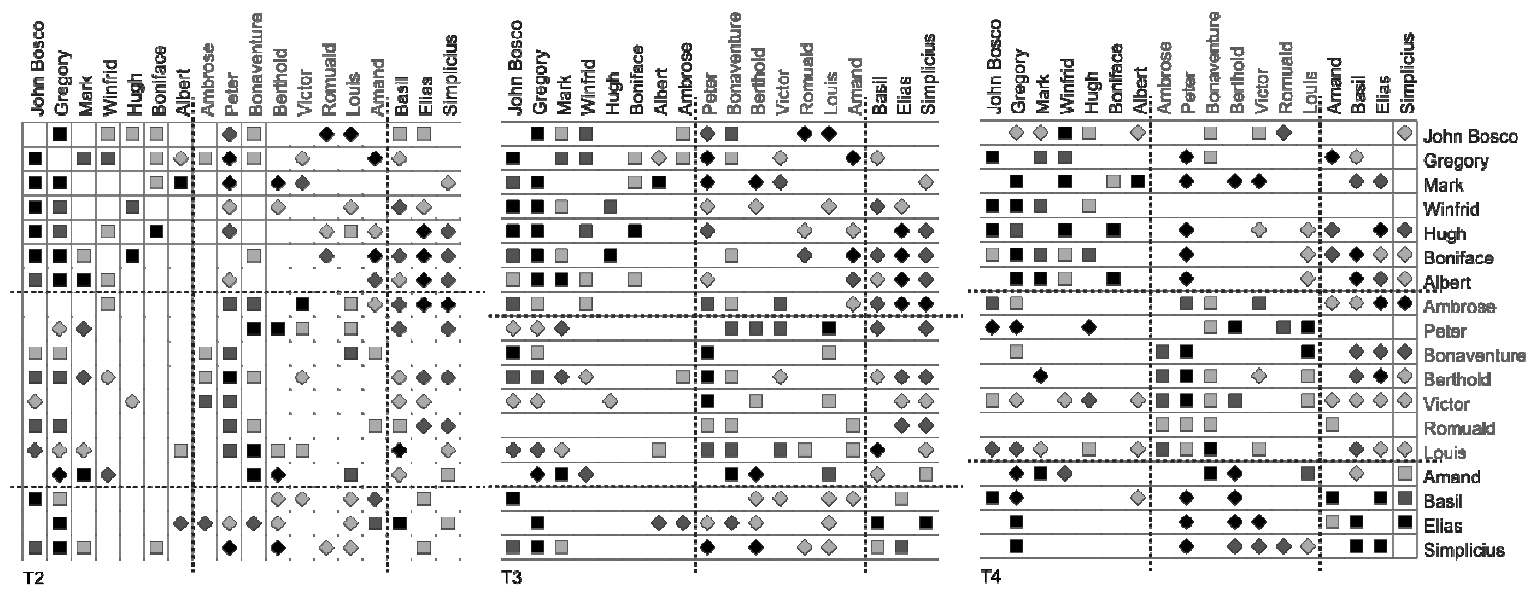

Figure 5: Structural Balance Partitions for the Sampson data at each time point.

Figure 6 shows the relaxed balance model as fitted for each time point with $k=$ 4. For $\mathrm{T}_{2}$, the Loyal Opposition has been split into two clusters. Four of their members (Bonaventure, Berthold, Ambrose and Romuald) send mainly positive ties to members of the Young Turks, a feature obscured in the structural balance partition. Consistent with structural balance, they send positive ties to others in the Loyal Opposition and negative ties to those in the Outcasts. The two partitions at $\mathrm{T}_{3}$ differ only in the location of Albert, again with positive blocks off the main diagonal. At $\mathrm{T}_{4}$, Bonaventure and Ambrose form a single cluster, receiving positive ties from members of the other three clusters. They also have reciprocated

28 Doreian and Mrvar (1996) had Amand with the Outcasts at all three time points. 
positive ties. This column of positive blocks supports the Sampson data conforming to the SB_DP model at $\mathrm{T}_{4}$.
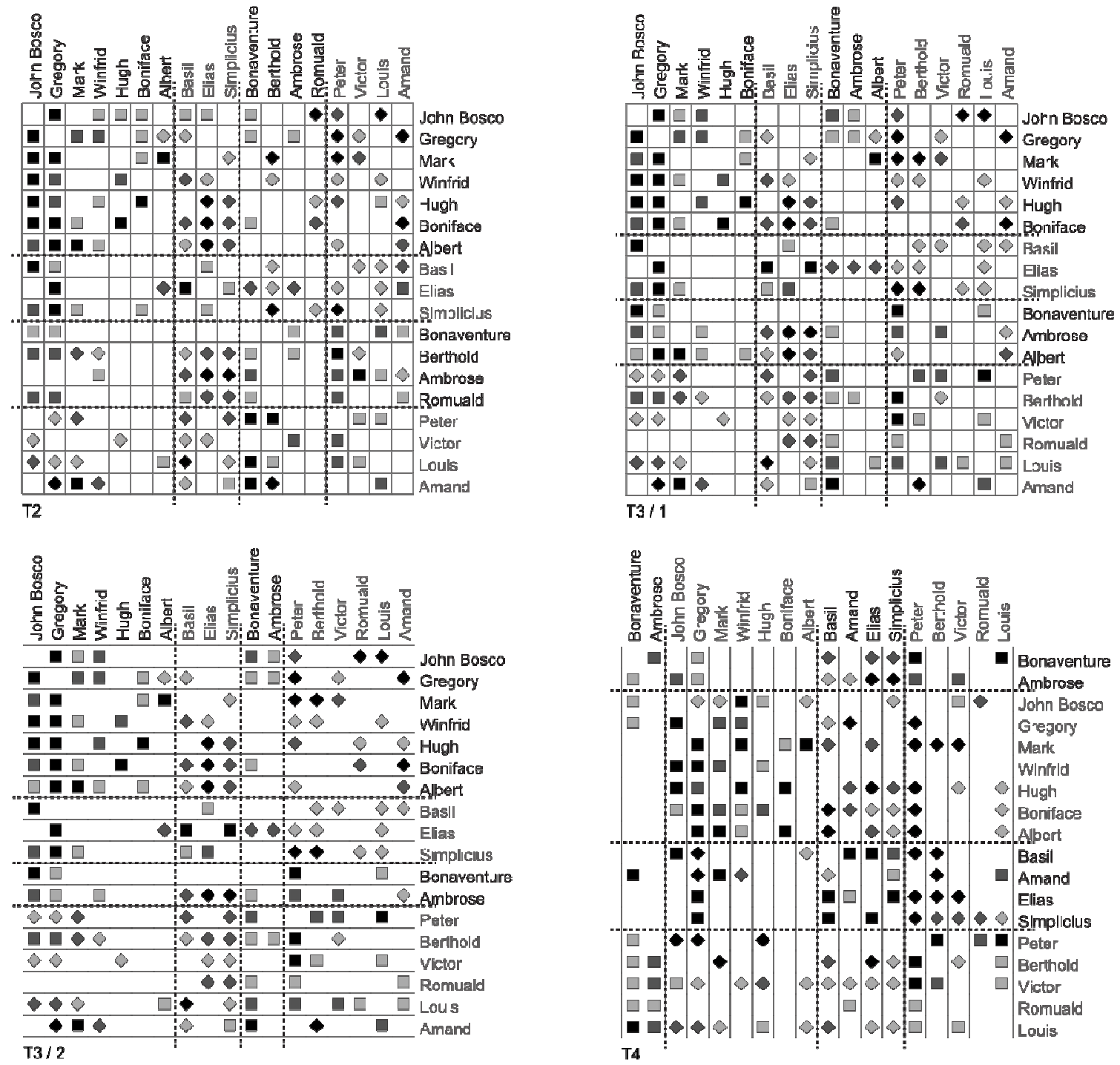

Figure 6: Relaxed Balance Blockmodels for the Sampson data at each time point $(k=4)$.

Figure 7 shows plots of inequality in the receipt of positive and negative ties. Consistent with the Newcomb data results, inequality of the receipt of negative ties increases across all time points. The pattern for inequality in the receipt of positive ties differs. From $\mathrm{T}_{2}$ to $\mathrm{T}_{3}$, it drops slightly before a sharp increase between $T_{3}$ and $T_{4}$. The highest value for each index is at $T_{4}$ providing support for Hypothesis 3 for the receipt of negative ties but only partial support for the receipt of positive ties. 


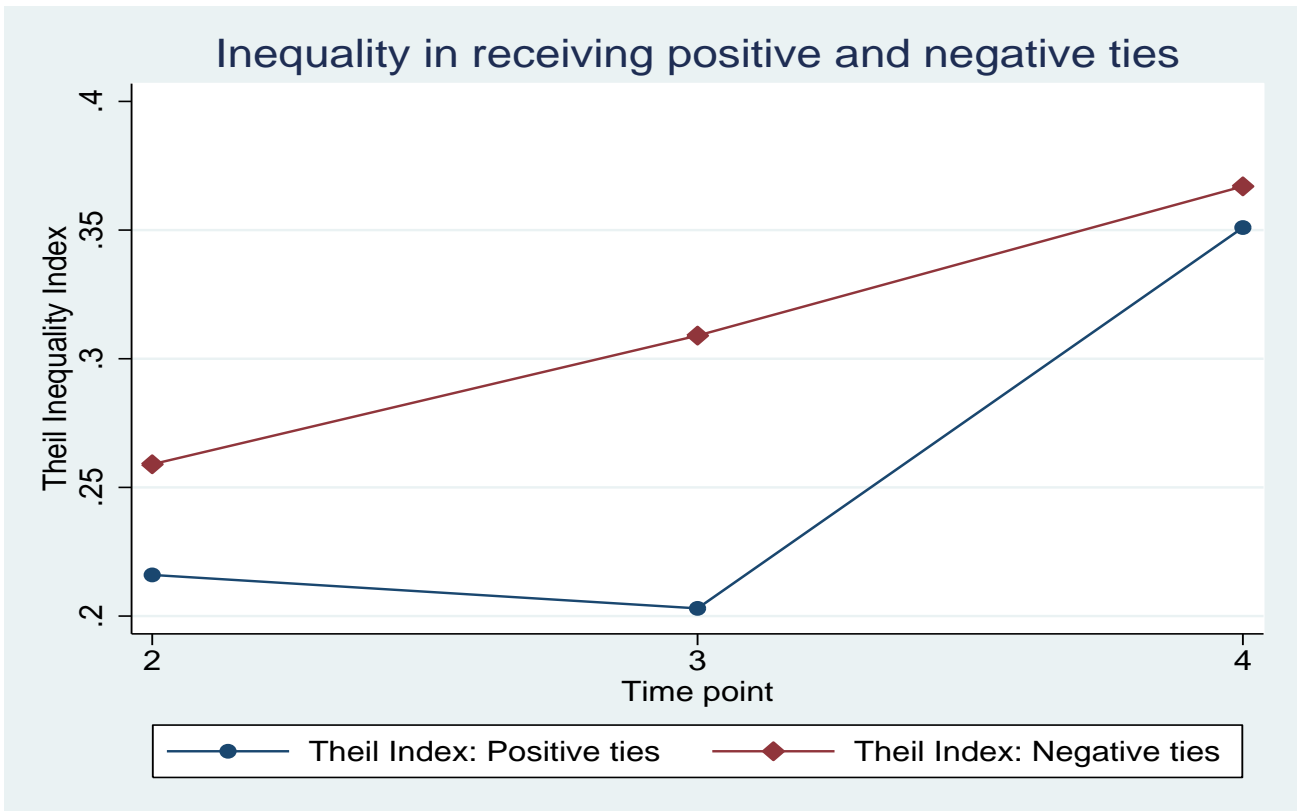

Figure 7: Inequalities in receiving positive and negative ties: Sampson data.

\section{Summary and discussion}

As multiple processes generate social relations among human actors, it is problematic to commit to examining only one process. The relevant processes include structural balance, differential popularity, differential dislike, and mutual hostility within subgroups larger than dyads. When processes operate they leave traces as structural features of networks. Our attempt to disentangle the results of these processes focused on the structure of the network as represented by locations of positive and negative block types in blockmodels. We used the generalized blockmodel of relaxed structural balance (Doreian and Mrvar, 2009) to fit blockmodels to signed networks. We found strong support for the operation of differential popularity in a column of off-diagonal positive blocks with the Newcomb data. Some actors in were universally popular, contrary to structural balance. Evidence was found also of subgroups of mutually hostile actors with persistent negative blocks on the main diagonal of the image matrix, also contradictory of structural balance.

The persistent presence of a column of off-diagonal negative blocks is consistent with both structural balance and differential dislike. By considering the increased concentration of negative blocks over time on a subset of actors, we infer that differential dislike contributes more than structural balance even though the results of these processes could not be disentangled completely. The results were less clear for the Sampson data where the structural balance model fared less badly than in the Newcomb data. There was some modest evidence for a weaker 
form of a model with differential popularity. Only at the last time point, in a model with four positions, was differential popularity more evident.

As a summary, in Newcomb's data, relaxed structural balance partitions provide strong support for the operation of differential capturing structural features at odds with structural balance. The increased concentration of negative ties on some actors suggests differential dislike is either a more potent process than structural balance or is an unrecognized component of it. The evidence for such outcomes was not as clear with Sampson's data. Yet there was support for the hypothesis regarding inequalities in receiving negative ties.

There are some caveats concerning our results because the data we used are not ideal. The recoding of Newcomb's data, used by others before us imposes the equivalent of a fixed choice design and is, at most, only an approximation of satisfactory temporal signed network data. Sampson also adopted a fixed choice design for the data he collected. Neither Newcomb's nor Sampson's data have systematic information regarding actor attributes. This imposes another limitation. Increasing concentration of receiving both positive and negative ties could rest on clearer perceptions of actor attributes and the accumulation of network processes. Without information on actor attributes and the recognition of this information by actors when forming and breaking signed ties these two processes cannot be disentangled. Some implications of these limitations are clear.

First, better over time network data for signed (and unsigned) networks in small groups are needed. Second, as networks and actors co-evolve, we need actor attribute data and (changing) actor perceptions of each other. Third, an adequate theory of network change requires reconsidering Heider's (1946) distinction between signed social relations and unit formation relations to incorporate both when studying actor and network co-evolution ${ }^{29}$. Using only structural (network) data is not enough. Even so, we have shown that network processes can be disentangled to some extent by delineating the structural traces that their operation leaves behind. This allowed for some comparative testing of theories about generating structures.

Such an approach can be made more fruitful by embedding signed blockmodeling in a richer substantive framework with more complete data. Here, we have written about tie formation without being attentive to the micro-processes involved for pairs of actors. Montoya and Insko (2008) analyze reciprocity in terms of affective, cognitive, and behavioral elements. Wojciszke et al. (2009) examine different mechanisms generating like-dislike and respect-disrespect relations. However these mechanisms operate, they will be constrained to some extent by the macro structure of the group within which they operate. It suggests also that a more general account will emerge from combining these different approaches. 29 White (1979) notes empirical evaluations of balance theory differ according to whether poq-
triples or pox-triples (with unit formation ties) are used. 
Another item meriting attention comes from the differences between the two sites where Newcomb and Sampson collected their data. The students in the pseudo-fraternity of Newcomb had potential relations and contacts outside their residential hall. In contrast, the trainee monks were largely cut off from the outside world. Such differences could make a difference in the macro network structures formed (Doreian and Conti, 2012). In terms of substance, theories of how relational tie formation is dependent on the context within which relations are formed are needed for a better account of the processes of network formation and the resulting network structures.

Another very promising approach to social networks are exponential random graph models (ergms). It would seem useful to explicitly couple the micro-process generation of network structure represented in the use of dynamic exponential random graph models with the kind of block modeling approach used here. We think that coupling the ergm approach to block modeling is an step. The simplest way of doing this is to incorporate block structures as a covariate. Doreian and Conti (2012) provide an example where both estimated ergm parameters and a blockmodel covariate were significant. A much deeper approach is to develop an ergm and a blockmodel simultaneously.

We provide a different take on two classical data sets by using signed blockmodeling to comparatively assess two theories about the generation of structure. However, we are mindful that these data sets are unique and imply some problems with regard to generalization, especially to larger networks. Balance theoretic ideas were formed in the study of small networks but it is reasonable to anticipate their extension to larger signed networks where overall network density tends to be lower. This raises the issue of whether density could affect the use of relaxed structural balance and structural balance. We think this would not affect our methods, especially if fixed choice designs are avoided. However, this remains an empirical issue. In terms of formal analysis, Abell and Ludwig (2009) have launched a program of research based on simulation studies of balance processes in larger signed networks Their simulated networks are very dense and, while they are useful for studying the operation of balance processes, it is not clear that there is a direct extension to empirical signed networks.

If areas of differential density exist in large signed networks, then the empirical study of large 'patchy' signed networks could benefit from the kinds of community detection methods developed by Traag and Bruggeman (2009) for signed networks. We provide a methodological comparison of this algorithm with RSB in the Appendix A. For the Newcomb data, the results are mixed but point to the RSB approach as more useful. The criterion functions implied by the two algorithms are different and it may be useful in future work to try and combine them in some fashion. Having diagonal blocks with dense positive lines seems important provided that this does not destroy the block structures identified here. 


\section{References}

[1] Abell, P. and Ludwig, M. (2009): Structural balance, a dynamic perspective, Journal of Mathematical Sociology, 33, 1-27.

[2] Abelson, R.P., McGuire, W.J., Newcomb, T.E., Rosenberg, M.J., and Tannenbaum, P.H. (Eds.) (1968): Theories of Cognitive Consistency: A Sourcebook, Chicago: Rand-McNally.

[3] Batagelj, V. and Mrvar, A. (1998): Pajek program for large network analysis, Connections, 21(2), 47-57.

[4] Borgatti, S.P. and Everett, M.G. (1999): Models of core/periphery structures, Social Networks, 21, 375-395.

[5] Borgatti, S.P., Everett, M.G., and Freeman, L.C. (2002): UCInet for Windows: Software for Social Network Analysis. Harvard, MA: Analytic Technologies.

[6] Breiger, R.L., Boorman, S.A., and Arabie, P. (1975): An algorithm for clustering relational data with applications to social network analysis and comparison to multidimensional scaling, Journal of Mathematical Psychology, 12, 328-383.

[7] Brusco, M.J., Doreian, P., Mrvar, A., and Steinley, D. (2011): Two algorithms for relaxed structural balance partitioning: Linking theory, methods and data to understand social network phenomena, Sociological Methods and Research, 40, 57-87.

[8] Cartwright, D. and Harary, F. (1956): Structural balance: A generalization of Heider's theory, Psychological Review, 63, 277-292.

[9] Davis, J.A. (1967): Clustering and structural balance in graphs, Human Relations, 20, 181-187.

[10] Davis, J.A and Leinhardt, S. (1972): The structure of positive interpersonal relations in small groups, 218-251. In Berger, J., Zelditch Jr., M. and Anderson, B.(Eds.): Sociological Theories in Progress Volume 2, , Boston: Houghton Mifflin.

[11] Dekker, D., Krackhardt, D., and Snijders, T.A.B. (2007): Sensitivity of MRQAP tests to collinearity and autocorrelation conditions, Psychometrika, 72, 563-581.

[12] Doreian, P. (2008): A multiple indicator approach to structural balance, Social Networks, 30, 247-258.

[13] Doreian, P., Batagelj, V., and Ferligoj, A. (2005): Generalized Blockmodeling. New York: Cambridge University Press.

[14] Doreian, P., Batagelj, V., and Ferligoj, A. (2004): Generalized blockmodeling of two-mode network data, Social Networks, 26, 29-53.

[15] Doreian, P. and Conti, N. (2012): Social infrastructure, geography and social network structure, Social Networks, 34, 32-46. 
[16] Doreian, P., Kapuscinski, R., Krackhardt, D., and Szczypula, J. (1996): A brief history of balance through time, Journal of Mathematical Sociology. 21, 113131 (reprinted in Evolution of Social Networks, Doreian, P. and Stokman, F. N. (Eds.) (pp 129-147), New York: Gordon and Breach.)

[17] Doreian, P. and Mrvar, A. (2009): Partitioning signed social networks, Social Networks, 31, 1-11.

[18] Doreian, P. and Mrvar, A. (1996): A partitioning approach to structural balance, Social Networks. 18, 149-168.

[19] Feld S.L. and Elsmore, R. (1984): Patterns of sociometric choices: Transitivity reconsidered, Social Psychology Quarterly, 45, 77-85.

[20] Ferligoj, A., Doreian, P., and Batagelj, V. (2011): Positions and roles. In Scott, J. and Carrington, P.J. (Eds.): Social Network Analysis. 434-446 Los Angeles: Sage.

[21] Festinger, L. (1957): A Theory of Cognitive Dissonance, Evanston: Row, Peterson.

[22] Hallinan, M.T. (1984): Cognitive balance and differential popularity in social networks. Social Psychology Quarterly, 45, 86-90.

[23] Harary, F., Norman, R. Z. and Cartwright, D. (1965): Structural Models. New York: John Wiley and Sons.

[24] Heider, F. (1946): Attitudes and cognitive organization, Journal of Psychology. 21, 107-112.

[25] Heider, F. (1958): The Psychology of Interpersonal Relations. New York: Wiley

[26] Holland, P. and Leinhardt, S. (1973): The structural implications of measurement error in sociometry, Journal of Mathematical Sociology, 3, 85112.

[27] Holland, P. and Leinhardt, S. (1972): Some evidence on the transitivity of positive interpersonal sentiment, American Journal of Sociology. 72, 12051209.

[28] Hummert, M.L., Crockett, W.H., and Kemper, S. (1990): Processing mechanisms underlying use of the balance schema, Journal of Personality and Social Psychology, 58, 5-21.

[29] Hummon, N.P. and Doreian, P. (2003): Some dynamics of social balance processes: Bringing Heider back into balance theory, Social Networks. 25, 1749.

[30] Leicht, E.A. and Newman, M.E.J. (2008): Community structure in directed networks, Physical Review Letters, 100, 118703.

[31] Leik, R.K. and B.F. Meeker (1975): Mathematical Sociology. Englewood Cliffs, NJ: Prentice Hall. 
[32] Montoya, R.M. and Insko, C.A. (2008): Towards a more complete understanding of the reciprocity of liking effect, European Journal of Social Psychology, 38, 477-498.

[33] Mower White, C.J. (1979): Factors affecting balance, agreement and positivity biases in POQ and POX triads, European Journal of Social Psychology, 7, 129-148.

[34] Newcomb, T.M. (1961): The Acquaintance Process. New York: Holt, Rinehart, \& Winston.

[35] Newman, M.E.J. (2006): Modularity and community structure in networks, Proceedings of the National Academy of Sciences (USA), 103 (23), 85778582.

[36] Nordlie, P. (1958): A Longitudinal Study of Interpersonal Attraction in a Natural Setting, Unpublished Ph. D. Dissertation, University of Michigan.

[37] Osgood, C.E. and Tannenbaum, P.H. (1955): The principle of congruity in the prediction of attitude change, Psychological Review, 62, 42-55.

[38] Robins, G. and Kashima, Y. (2008): Social psychology and social networks: Individuals and social systems, Asian Journal of Social Psychology, 11, 1-12.

[39] Sampson, S.F. (1968): A Novitiate in a Period of Change: An Experimental Case Study of Relationships, Unpublished Ph.D. Dissertation, Department of Sociology, Cornell University, Ithaca, NY.

[40] Taylor, H.F. (1970): Balance in Small Groups. New York: Van Nostrand Reinhold.

[41] Theil, H. (1967): Economics and Information Theory. Chicago: Rand McNally.

[42] Traag, V.A. and Bruggeman (2009): Community detection in networks with positive and negative links, arXiv:0811.2329v3 [physics.soc=ph], 25 September, 2009.

[43] Wasserman, S. and Faust, K. (1994): Social Network Analysis: Methods and Applications. Cambridge: Cambridge University Press.

[44] Wojciszke, B., Abele, A.E., and Baryla, W. (2009): Two dimensions of interpersonal attitudes: Liking depends on communion, respect depends on agency, European Journal of Social Psychology, 39, 973-990.

\section{Appendix A}

Another approach to partitioning networks exists within the community detection literature. Community detection and blockmodeling are two methods for partitioning social networks developed separately but with obvious parallels. In order to compare them, the algorithm of Traag and Bruggeman (2009), devised specifically for signed networks, is best placed for this. It has been implemented in 
pajek (Batagelj and Mrvar, 1998). The algorithm is based on an adaptation of modularity (Newman, 2006; Leicht and Newman, 2008) and maximizes positive and minimizes negative lines within diagonal blocks while it minimizes positive and maximizes negative lines in off-diagonal blocks. In using this approach, we obtained higher values of the modularity index for partitions having a high density of positive and low density of negative lines inside clusters and a high density of negative and low density of positive lines between clusters.

We note that partitioning signed networks using relaxed structural balance (RSB) is driven by substance concerning the dynamics of relations in small groups while community detection is driven more by the observation that communities have denser positive ties and sparser (or even no) negative ties within them compared to the ties to the rest of the network. It is useful for partitioning large networks. It is reasonable to compare them.

This comparison is purely methodological and takes the following form: i) produce the best partitions using the Traag and Bruggeman algorithm; ii) establish the corresponding RSB partitions (with the same values of $k$ ); iii) create the implied fitted matrix arrays for both; iv) establish how well they predict the actual data; and v) compare the two partitions in relation to each other. The results are shown in Table A.1: the first column lists time points; the second column has the number of positions (clusters) obtained by the community detection (CD) algorithm and used also for the corresponding RSB partitions; the third column has the variance explained by the community detection partitions; the fourth column has the variance explained by the RSB partitions; and the final column has a direct comparison of the pairs of fitted partitions. The comparison is made solely in terms of the number of clusters determined CD and defers to these values of $k$. The result is straightforward: at each time point the variance explained by the RSB approach is larger than the variance explained by community detection. However, for four time points the differences are trivially small and a reasonable conclusion is that the two partitions perform equally well in predicting the empirical relational arrays for these time points. Thereafter, in contrast, the differences are more substantial and sometimes the differences are large. We note that the correlations between the two fitted arrays are particularly high for $t_{4}$ and $t_{7}$. The variation of $R^{2}$ across the time points has more to do with the number of clusters: other things equal, using more positions leads to explaining more variance in the array of signed ties. Given that there are only 17 data points, even using 5 or 6 positions seems excessive. Using $k=4$ for all time points, as done in the paper, seems preferable both in terms of substance and for uniform comparisons.

For the primary substantive concerns considered here, the results of using the signed community detection approach are mixed. For five time points $\left(t_{2}, t_{3}, t_{4}, t_{5}\right.$, and $t_{10}$ ) there is no column of positive blocks. However, for the remaining times points, there is as least one column of positive blocks. This provides support for the SB_DP Model. Using this community detection algorithm permits a comparative test precluded by classical structural balance. For all time points, 
there are no diagonal negative blocks in the blockmodels obtained by the community detection approach: The presence of such blocks is missed and precluded the delineation (and examination) of the SB_DP_MD Model. We return to Leik and Meeker's point: coherence between substance, method, and data is important. The substantively driven RSB approach has this coherence while the community detection approach used here does not.

Table A.1: Comparing the predictive value of two partitions.

\begin{tabular}{|l|l|l|l|l|}
\hline Time & $\mathrm{k}$ & $\mathrm{R}^{2}$ & $\mathrm{R}^{2}$ & $\mathrm{R}^{2}$ \\
$(\mathrm{R})$ & $(\mathrm{CD})$ & $\left(\mathrm{CD} \_\mathrm{RSB}\right)$ \\
\hline $\mathrm{t}_{1}$ & 3 & 0.27 & 0.33 & 0.22 \\
$\mathrm{t}_{2}$ & 3 & 0.32 & 0.35 & 0.28 \\
$\mathrm{t}_{3}$ & 3 & 0.35 & 0.41 & 0.50 \\
$\mathrm{t}_{4}$ & 3 & 0.42 & 0.46 & 0.86 \\
$\mathrm{t}_{5}$ & 3 & 0.30 & 0.53 & 0.34 \\
$\mathrm{t}_{6}$ & 5 & 0.38 & 0.57 & 0.53 \\
$\mathrm{t}_{7}$ & 6 & 0.81 & 0.93 & 0.87 \\
$\mathrm{t}_{8}$ & 5 & 0.51 & 0.90 & 0.48 \\
$\mathrm{t}_{9}$ & 4 & 0.28 & 0.75 & 0.30 \\
$\mathrm{t}_{10}$ & 4 & 0.29 & 0.74 & 0.30 \\
$\mathrm{t}_{11}$ & 5 & 0.66 & 0.90 & 0.64 \\
$\mathrm{t}_{12}$ & 5 & 0.66 & 0.90 & 0.69 \\
$\mathrm{t}_{13}$ & 4 & 0.64 & 0.78 & 0.64 \\
$\mathrm{t}_{14}$ & 5 & 0.75 & 0.90 & 0.72 \\
$\mathrm{t}_{15}$ & 4 & 0.42 & 0.84 & 0.40 \\
\hline
\end{tabular}

CD - Community detection, RSB - Relaxed Structural Balance

\section{Appendix B}

All of the data analyses were done using three programs. The temporal plots in Figures 1, 3 and 4 were drawn using STATA. The fitting of blockmodels was done using Pajek (Batagelj and Mrvar, 1998) using pre-specified models. The commands for this are explained in the Pajek manual. The QAP regressions were 
done by using UCINET (Borgatti et al., 2002). The Pajek files for doing this were imported into UCINET. Again, using QAP is documented in the manual for this suite of programs. 